\title{
FOLFOXIRI als Alternative
}

FOLFIRINOX (5-Fluorouracil, Irinotecan, Oxaliplatin, Gemcitabin, nab-Paclitaxel) ist eine wirksame Erstlinientherapie bei fortgeschrittenem Pankreaskarzinom, die allerdings auch Toxizitäten auslöst. Nun wurde ein modifiziertes Therapieregime untersucht: Das FOLFOXIRI-Regime der Gruppo Oncologico Nord Ovest (GONO) enthält Irinotecan, Oxaliplatin, Folinsäure und 5-Fluorouracil.

In die Beobachtungsstudie wurden 137 Patienten mit fortgeschrittenem Pankreaskarzinom aufgenommen, die im Universitätsklinikum Pisa mit FOLFOXIRI behandelt wurden. Die ersten 78 Patienten erhielten ein modifiziertes FOLFOXIRI-Regime in leicht reduzierten Dosierungen, alle weiteren Patienten das klassische GONO-FOLFOXIRI-Regime.

Nach einer medianen Beobachtungsdauer von 30 Monaten lag das mediane progressionsfreie Überleben bei 8,0 Monaten und das mediane Gesamtüberleben bei 12 Monaten.
In einer multivariaten Analyse waren folgende Faktoren mit einem schlechteren Gesamtüberleben assoziiert: Lebermetastasen (Hazard Ratio [HR] 0,59; $\mathrm{p}=0,019)$, ein ECOG-Performanceatatus von 1 (HR 2,26; $\mathrm{p}=0,001)$ und ein Neutrophilen-Lymphozyten-Verhältnis $>4$ (HR 2,42; $\mathrm{p}=0,002)$. Anhand dieser Faktoren wurden 119 Patienten, für die vollständige Daten vorlagen, in Gruppen mit guter Prognose (kein prognostisch ungünstiger Faktor, 38 Patienten), mittlerer Prognose (1 prognostisch ungünstiger Faktor, 49 Patienten) und schlechter Prognose (2 oder 3 prognostisch ungünstige Faktoren, 32 Patienten) eingeteilt. Das mediane Gesamtüberleben betrug in diesen drei Gruppen 17,6, 11,1 bzw. 7,4 Monate $(\mathrm{p}<0,001)$.

Judith Neumaier

Vivaldi C et al. First-line treatment with FOLFOXIRI for advanced pancreatic cancer in clinical practice: Patients' outcome and analysis of prognostic factors. Int J Cancer. 2016;139(4):938-45.

\section{Hirnmetastasen}

\section{Am besten nur stereotaktisch bestrahlen}

Die alleinige stereotaktische Strahlenchirurgie kann bei Patienten mit einer bis drei Hirnmetastasen als bevorzugte Behandlungsmethode empfohlen werden, so das Fazit einer aktuellen Studie. 213 Patienten mit Hirnmetastasen einer malignen Erkrankung wurden auf stereotaktische Strahlenchirurgie allein oder in Kombination mit einer Ganzhirnbe-

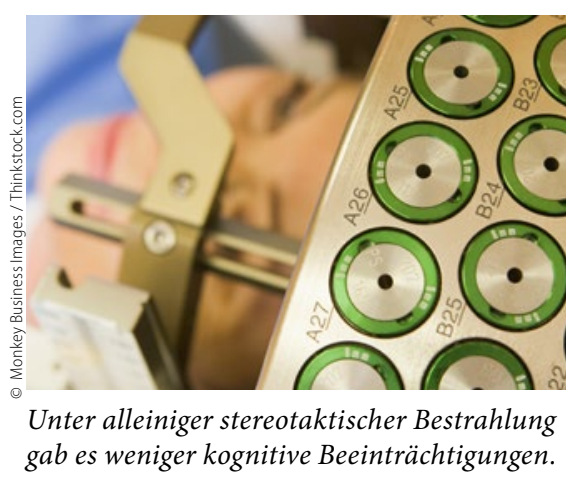

strahlung randomisiert. Das Durchschnittsalter betrug 60,6 Jahre.

Nach alleiniger stereotaktischer Strahlenchirurgie gab es nach drei Monaten weniger kognitive Beeinträchtigungen $(63,5 \%)$ als bei kombinierter stereotaktischer Bestrahlung und Ganzhirnbestrahlung (91,7\%; p < 0,001). Auch bei den Langzeitüberlebenden waren die kognitiven Beeinträchtigungen in der Gruppe mit alleiniger Stereotaxie nach drei Monaten (45,5 vs. $94,1 \%$; $=0,007)$ und nach zwölf Monaten (60 vs. 94,4\%; $\mathrm{p}=$ $0,04)$ geringer.

Bezüglich funktionaler Unabhängigkeit gab es nach drei Monaten zwischen den Therapiegruppen keinen signifikanten Unterschied (mittlere Veränderung $-1,5$ vs. $-4,2$ Punkte; $p=0,26)$. Die Lebensqualität war bei alleiniger stereotaktischer Strahlenchirurgie nach drei Monaten besser, darunter die generelle Lebensqualität (mittlere Veränderung von

\section{kurz notiert}

Forschungsstandort Deutschland

SPARCL1 hemmt Angiogenese +++

Wissenschaftler der Universität Erlangen-Nürnberg stellten fest, dass Endothelzellen aus Tumoren von Patienten mit guter Prognose das Protein SPARCL1 expremieren, das sowohl das Wachstum von Endothelzellen als auch von Tumorzellen hemmt. Bei Tumoren mit schlechter Prognose hingegen wurde das Protein nicht exprimiert, wurden neue Blutgefäße gebildet und der Tumor wuchs weiter [Naschberger E et al. J Clin Invest. 2016;126(11):4187-204]. red.

Therapiekontrolle durch Liquid Biopsy +++ Die Liquid Biopsy ist eine vielversprechende Methode zur frühen Therapiekontrolle bei Lungenkrebspatienten. Dabei wird im Blut zellfreie Tumor-DNA nachgewiesen. Heidelberger Wissenschaftler konnten damit in Echtzeit verfolgen, wie Tumoren auf die Therapie ansprachen. Sie zeigten, dass das Verfahren sensitiv genug ist, um Tumorveränderungen in Echtzeit nachzuweisen. Gleichzeitig hat die Methode den Vorteil, dass sie weniger invasiv als eine Gewebeentnahme ist. Die so gewonnenen molekularen Daten könnten auch Informationen liefern, die es erlauben, frühzeitig über geeignete therapeutische Strategien zu entscheiden [Riediger AL et al. Sci Rep. 2016;6:33505].

red.
$-0,1$ vs. -12 Punkte; mittlere Differenz $11,9 \% ; \mathrm{p}=0,001)$.

Die Zeit bis zum intrakraniellen Rezidiv war bei alleiniger stereotaktischer Strahlenchirurgie signifikant kürzer als bei zusätzlicher Ganzhirnbestrahlung (Hazard Ratio [HR] 3,6). Zudem war die Rate der intrakraniellen Krankheitskontrolle nach drei Monaten in der Gruppe mit Stereotaxie plus Ganzhirnbestrahlung signifikant besser als in der Gruppe mit alleiniger stereotaktische Strahlenchirurgie ( 93,7 vs. $75,3 \%$ ), ebenso nach sechs und zwölf Monaten.

Doch trotz besserer Krankheitskontrolle bei Ganzhirnbestrahlung gab es keine Unterschiede im Gesamtüberleben: Bei alleiniger stereotaktischer Strahlentherapie wurde ein Gesamtüberleben von 10,4 Monaten, bei Stereotaxie plus Ganzhirnbestrahlung ein Gesamtüberleben von 7,4 Monaten (HR 1,02; p = 0,92) erreicht.

Kathrin von Kieseritzky

Brown PD et al. Effect of Radiosurgery Alone vs Radiosurgery With Whole Brain Radiation Therapy on Cognitive Function in Patients With 1 to 3 Brain Metastases. JAMA. 2016;316(4):401-9. 